This is the author's final, peer-reviewed manuscript as accepted for publication. The publisher-formatted version may be available through the publisher's web site or your institution's library.

\title{
Measuring children's self-efficacy and proxy efficacy related to fruit and vegetable consumption
}

Karly S. Geller, David A. Dzewaltowski, Richard R. Rosenkranz, Konstantinos Karteroliotis

\section{How to cite this manuscript}

If you make reference to this version of the manuscript, use the following information:

Geller, K.S., Dzewaltowski, D.A., Rosenkranz, R.R., \& Karteroliotis, K. (2009). Measuring children's self-efficacy and proxy efficacy related to fruit and vegetable consumption. Retrieved from http://krex.ksu.edu

\section{Published Version Information}

Citation: Geller, K.S., Dzewaltowski, D.A., Rosenkranz, R.R., \& Karteroliotis, K. (2009). Measuring children's self-efficacy and proxy efficacy related to fruit and vegetable consumption. Journal of School Health, 79(2), 51-57.

Copyright: (C 2009, American School Health Association

Digital Object Identifier (DOI): doi: 10.1111/j.1746-1561.2008.00376.x

Publisher's Link: http://onlinelibrary.wiley.com/doi/10.1111/j.1746-1561.2008.00376.x

This item was retrieved from the K-State Research Exchange (K-REx), the institutional repository of Kansas State University. K-REx is available at http://krex.ksu.edu 


\title{
Measuring Children's Self-Efficacy and Proxy Efficacy Related to Fruit and Vegetable Consumption
}

Geller, K.S., Dzewaltowski, D.A., Rosenkranz, R.R., \& Karteroliotis, K.

Suggested Citation: Geller, K.S., Dzewaltowski, D.A., Rosenkranz, R.R., \& Karteroliotis, K. (2009). Measuring children's self-efficacy and proxy efficacy related to fruit and vegetable consumption. Journal of School Health, 79(2):51-57.

The definitive version is available at www.blackwell-synergy.com or

http://onlinelibrary.wiley.com/doi/10.1111/j.1746-1561.2008.00376.x/full

Keywords: nutrition and diet; after-school; self-efficacy; fruit and vegetable

\begin{abstract}
BACKGROUND: Social cognitive theory describes self-efficacy and proxy efficacy as influences on fruit and vegetable consumption (FVC). Proxy efficacy was defined as a child's confidence in his or her skills and abilities to get others to act in one's interests to provide fruit and vegetable (FV) opportunities. The purpose of this study was to develop a scale assessing children's self-efficacy and proxy efficacy for FVC at after-school programs and at home.

METHODS: Elementary-aged children $(n=184)$ attending 7 after-school programs completed a self-efficacy questionnaire relevant to FVC. Questionnaire validity was investigated with exploratory factor analysis and mixed-model analysis of covariance. Internal consistency reliability and readability were also assessed.

RESULTS: The questionnaire assessed 4 constructs: self-efficacy expectations for fruit consumption, self-efficacy expectations for vegetable consumption, proxy efficacy to influence parents to make FV available, and proxy efficacy to influence after-school staff to make FV available. Children perceiving FV opportunities in after-school had greater self-efficacy expectations for FVC and greater proxy efficacy to influence after-school staff compared to students who did not perceive FV opportunities. Children attending schools of higher socioeconomic status (SES) and less diversity were more confident they could influence their parents to make FV available than students attending lower SES and
\end{abstract}


less diverse schools. Adequate internal consistency and test-retest reliabilities were established.

CONCLUSIONS: Self-efficacy is a multicomponent construct that can be assessed in children using the reliable and valid instrument evaluated by the current study.

Social-cognitive theory (SCT) is one of the predominant models for understanding and impacting health behaviors, having been applied in several studies investigating psychosocial influences on fruit and vegetable consumption (FVC). One influence identified by SCT is self-efficacy, which is defined as a child's belief that he or she can execute a behavior at a level necessary to obtain a desired outcome. ${ }^{1}$ Several studies have shown that self-efficacy influences FVC in elementary ${ }^{2-5}$ and middle school youth. ${ }^{6}$

Self-efficacy reflects two ways of reaching a desired outcome: direct personal agency and proxy agency. ${ }^{7}$ Direct personal agency has been assessed by having children estimate their confidence in eating fruit and vegetables (FV). Proxy agency is reflected in this self-efficacy judgment, but it can also be assessed directly by measuring children's proxy efficacy. Proxy efficacy is the belief that one can get others to act on their behalf to reach desired outcomes. ${ }^{7}$

Because children are not directly in charge of the social and institutional practices that provide FV opportunities in their environments, they may need to exert proxy efficacy. ${ }^{8}$ When children's proxy efficacy is high, they are more likely to request FV from others they perceive to be proficient enough to act on their behalf. These proxy agency efforts may then result in increased FV opportunities, increased self-efficacy, and an increased likelihood of FVC.

Previous FV research has not adequately distinguished between self-efficacy and proxy efficacy in the measurement of these constructs. For example, Reynolds and colleagues $^{9}$ performed statistical analyses that revealed a single factor for self-efficacy, merging direct personal agency and proxy agency into one construct. However, the 21item self-efficacy questionnaire had 17-items probing children's perceptions of direct agency ("I can...") as well as 4-items investigating perceptions of proxy agency ("I can 
ask my mom or dad...”). Specific attention to the conceptual distinction between direct personal agency and proxy agency may reveal that these are separate but related constructs. The specific analytical plan and results for concluding that there was a onedimensional self-efficacy scale was not discussed in Reynolds and colleagues' paper. ${ }^{9}$ A plausible explanation may be the grouping of 17 direct personal agency items with only four proxy efficacy items resulted in weak factor separation.

The primary aim of the present study was to determine if self-efficacy and proxy efficacy could be measured with reliability and validity in late elementary school-aged children. Children's self-efficacy for FVC and proxy efficacy for FV opportunities were examined with a self-report questionnaire. The secondary aim was to investigate whether the present measures could detect differences in direct personal agency and proxy agency between groups of children that theoretically should differ (criterion validity). Group differences were examined between children who perceived FV opportunities afterschool compared to children who did not perceive FV opportunities and between afterschool children attending schools with higher concentrations of racial/ethnic diversity and higher concentrations of lower-socioeconomic status (SES) compared to lower concentrations of racial/ethnic diversity and higher concentrations of higher-SES.

Overall, the questionnaire was expected to emerge as multidimensional, containing both a self-efficacy scale and proxy efficacy scale. Additionally, the direct personal agency scale was expected to distinguish between direct personal agency for fruit and direct personal agency for vegetable consumption, coinciding with research reporting fruit consumption and vegetable consumption as two separate behaviors. ${ }^{10-12}$ Two separate subscales for proxy agency were also expected, one representing proxy agency from parents and the other proxy agency from after-school staff. Finally, the establishment of criterion validity was expected such that there would be differences between groups on the direct personal agency and proxy agency measures based on their perception of opportunities for FVC in their after-school programs and the diversity-SES classification of their school. It was hypothesized that children attending after school environments with greater opportunities for FVC would have higher self-efficacy and proxy efficacy compared to children attending after school environments with fewer opportunities. Also, it was hypothesized that children in high-resource environments 
(higher-SES schools) would have greater self-efficacy and proxy efficacy compared to children in low-resource environments (lower-SES schools).

\section{Methods}

\section{Subjects}

Participants were fourth-, fifth- and sixth-grade children recruited from seven after-school programs located in Lawrence Kansas. Children completed a 61-item questionnaire (approximately 30 minutes) regarding their physical activity and nutritional beliefs and behaviors. Of those enrolled in the after-school program, 74\% participated in fall 2005 and $70 \%$ in fall 2006. Some children participating in fall 2005 also completed the questionnaire in fall 2006, but were dropped from the fall 2006 database. The final database used for statistical analysis included 54\% of children surveyed in fall 2005 and $46 \%$ in fall 2006. Of the 187 children, 184 (98\%) had complete self-efficacy data (14items) and complete perceived opportunity for FV data (2 items). All demographic data (i.e., gender, age, lunch status/SES, and ethnicity) were obtained directly from school records.

The 184 children were among an after-school group primarily composed of fourth-graders, but containing other grades of similar age ( $8 \%$ fifth-grade, and $2 \%$ sixthgrade). The mean age during the time of questionnaire completion was 9 years, ranging between 8 and 12 years. Forty-seven percent of the sample was female and $41 \%$ was lower-SES (i.e., receiving free and reduced meal program assistance). The sample was primarily white $(n=131)$, with some diversity (Black, $n=29$; American Indian/Alaska native, $n=15$; Hispanic/Latino, $n=6$; Asian, $n=2$; Native Hawaiian/other, $n=1$ ).

\section{Procedure}

The current analysis drew data from the Healthy Opportunities for Physical Activity and Nutrition (HOP'N) project, a school-randomized controlled trial targeting the prevention of obesity. All data were collected during baseline prior to intervention from youth whose parents or guardians provided active informed consent. The Institutional Review Board (IRB) at Kansas State University approved all procedures. During after-school programs at seven elementary school sites, research assistants led 
groups of children through a paper-and-pencil survey assessing psychosocial variables related to physical activity and nutrition.

Using a verbatim script, all instructions and questions were simultaneously read aloud to all participating children. Children completed the questionnaire individually, but were asked to wait and follow along as a research assistant read each question aloud to all children in the class. The script included questionnaire instructions and definitions of FV serving sizes. Children were also shown realistic FV food models, functioning as visual aids that insured their understanding of FV serving sizes. Finally, a large poster board displaying written definitions and example questions was presented to the group. Following completion, all children who participated in the survey were privately given small incentives (i.e., colorful pencils, small toys); however, no penalty for nonparticipation was employed.

\section{Instruments}

\section{Direct Personal Agency and Proxy Agency Measures}

Four groups of items were developed by the research team based on SCT and FV literature. ${ }^{10-11}$ The construct of personal agency, labeled in this study as self-efficacy, was assessed with the first group of items $(\mathrm{n}=3)$ for both fruit consumption (SE-FRUIT) as well as a second group of items $(n=3)$ for vegetable consumption (SE-VEG). A third group of items $(n=4)$ captured proxy agency relevant to parents, which is referred to as proxy efficacy for FV availability from parents (PEFV-P). A final group of items $(n=4)$ captured proxy agency relevant to the after-school staff, labeled here as proxy efficacy for FV availability from staff (PEFV-S).

The SMOG test was chosen for performing readability tests on the entire 14-item questionnaire, as well as each of the four subscales. The SMOG readability analyses gave the 14-item questionnaire a seventh-grade score, and each subscale ranged from third- to eighth-grade $(\mathrm{SD}=+1.5)$. Although these grade-levels exceed that of the present subjects (fourth-, fifth-, and sixth-graders), it should be noted that all instructions and each individual question was read out-loud to the children before they responded. Furthermore, only six different polysyllabic words were included among the questionnaire items including: vegetable(s), favorite, refrigerator, banana, apricots and 
applesauce. These words, although polysyllabic, are usually highly recognizable by children when read aloud.

Self-Efficacy for Fruit Consumption (SE-FRUIT). The self-efficacy for fruit consumption items were generated to correspond to the recommendation of one to three servings of fruit or $100 \%$ fruit juice each day. ${ }^{13}$ Serving sizes were established from the food guide pyramid; therefore, one serving of fruit and one serving of fruit juice was defined to the children as " 1 medium piece of fresh fruit, 1/2 cup of fruit salad, $1 / 4$ cup of raisins, apricots or other dried fruit, 6 oz. of 100\% orange, apple or grape juice (Do not count fruit punch, lemonade, Gatorade, Sunny Delight or fruit drink)." Each question began with "How sure are you that you can eat," assessing in three separate questions confidence to eat one, two and three servings of fruit each day (Table 2.1). Children responded using a three-point scale, "Not sure at all," "Somewhat sure" and "Very sure."

Self-Efficacy for Vegetable Consumption (SE-VEG). Similar to SE-FRUIT, selfefficacy for vegetable consumption items were generated based on the food guide pyramid (one to three servings each day). ${ }^{13}$ One serving of a vegetable was defined for the children as " 1 medium carrot or other fresh vegetable, 1 small bowl of green salad, 1/2 cup of fresh or cooked vegetables, 3/4 cup of vegetable soup (Do not count French fries, onion rings, potato chips or fried okra)." These questions were grouped with fruit consumption items, beginning with "How sure are you that you can eat." Three separate questions were included assessing children's perceived ability to consume one, two and three servings of vegetables. Children responded using the same three-point scale ("Not sure at all," "Somewhat sure" or "Very sure)."

Proxy Efficacy for Fruit and Vegetables- Parent (PEFV-P). Proxy efficacy for FV availability was defined as children's confidence in their skills and abilities to get parents to make FV available. Specifically, PEFV-P assessed children's confidence in having a parent or guardian provide them with fruits, fruit juices, and vegetables (Table 2.1). An example question was, "How sure are you that you can get your parents to buy fruit for a snack." Children responded to each item using a three-point scale, "Not sure at all," "Somewhat sure" and "Very sure."

Proxy Efficacy for Fruit and Vegetables- Staff (PEFV-S). PEFV-S was defined as children's confidence in their skills and abilities to get the after-school program staff 
members to make fruit, fruit juice and vegetables available (Table 2.1). Similar to PEFVP, children responded to each item using a three-point scale, "Not sure at all," "Somewhat sure" and "Very sure." An example question was, "How sure are you that you can get the teachers or staff members of the after-school program to offer fruit and vegetable snack options."

\section{After-School Environment Measures}

Perceived School Fruit and Vegetable Opportunity. Two items assessed children's perceived opportunities for FV during the after-school program. Children responded on a three-point scale choosing among "yes," "don't know," or "no" to "There are a lot of chances to eat fruit and vegetables at the after-school program" and "We are satisfied with the fruits and vegetables offered at the after-school program." The sample of children whose response was "yes" was categorized as perceiving FV opportunities in after-school. Internal consistency of the two-item scale was 0.65 .

School Diversity and SES. Seven schools were grouped into two categories based on the percentage of youth qualifying for free and reduced lunch and percentage of youth who were white or of diverse race/ethnicity. The higher diversity and lower-SES schools $(n=4)$ ranged from $63 \%$ of the youth qualifying for free and reduced school meals to $89 \%$. These schools had approximately $50 \% \mathrm{racial} /$ ethnic diversity with one school having slightly lower diversity (28\%). The lower diversity and higher-SES schools $(n=3)$ ranged in free and reduced status from $32 \%$ to $4 \%$ and in diversity from $13 \%$ to $24 \%$.

\section{Data Analysis}

Exploratory Factor Analysis (EFA) was performed using SPSS 13.0 with principal axis factor extraction method, followed by direct oblique (oblimin) rotation. This rotation method was used due to hypothesized correlations among the underlying factor structures of self-efficacy. The number of factors retained was determined using the following criteria: (a) Factors with unrotated eigenvalues exceeding $1,{ }^{14}$ (b) a scree test, ${ }^{15}$ and (c) factor loadings exceeding $0.40 .{ }^{16}$ Item reliability was estimated with Cronbach's alpha $(\alpha)$ and equal-length Spearman-Brown correlation coefficients.

Criterion validity analyses were performed using SAS software (version 9.1; SAS Institute. Cary, NC). Differences in FV self-efficacy and proxy efficacy variables were 
evaluated for significance using a mixed-model analysis of covariance (PROC MIXED). To examine between group differences, the model included gender, ethnicity, household SES, and child weight status as fixed effects. Furthermore, children were nested within the after-school program as a random effect to address the possible clustering of children within any one of the seven after-school programs.

\section{Results}

\section{Exploratory Factor Analysis}

A principal axis factor (PAF) analysis of the 14 self-efficacy questionnaire items extracted four factors with eigenvalues greater than 1. In addition, a scree plot indicated the existence of four factors. The Keiser-Meyer-Olkin test of sampling adequacy coefficient was 0.76 , exceeding the 0.60 minimum required for factor analysis. Thus, the four-factor solution met all statistical criteria and accounted for approximately $68.1 \%$ of the variability among the 14 -items. Following oblique (oblimin) rotation, all items had factor loadings exceeding 0.40 on only one of the four identified factors, confirming the inclusion of all 14-items. Table 2.1 depicts the percent variance accounted for by each factor and the factor pattern coefficients for each item.

The first factor, labeled Self-Efficacy for Vegetable Consumption (SE-VEG), included three items capturing children's confidence in their ability to consume one, two and three servings of vegetables daily. Factor two was labeled Proxy Efficacy for Fruit and Vegetable Availability from After-School Staff (PEFV-Staff), and consisted of four items identifying children's perceptions of their ability to influence after-school staff members to make FV available. The third factor, labeled proxy efficacy for Fruit and Vegetable Availability from the Parent (PEFV-Parent), also consisted of four items and reflected children's perception of their ability to influence their parent(s) to make FV available. Finally, the fourth factor, labeled Self-Efficacy for Fruit Consumption (SEFRUIT) captured children's confidence in consumption of one, two and three servings of fruit.

Reliability of the questionnaire was quantified using all 184 child responses. There was high internal consistency for the entire 14-item questionnaire (Cronbach's Alpha $=0.81$ ), ranging between 0.75 and 0.84 for the four subscales. Additionally, split- 
half internal consistency method was employed to determine reliability. The reliability of the 14-item questionnaire was 0.56 (equal-length Spearman-Brown, $\mathrm{n}=184$ ). The coefficients of the four subscales were acceptably high, ranging between 0.74 and 0.80 .

\section{Criterion Validity}

Table 2.2 reports the group least squared means and standard errors. Group differences were found such that children perceiving FV opportunities during after-school were significantly greater in SE-FRUIT than children not perceiving these opportunities $(\mathrm{F}(1,176)=18.25, \mathrm{p}=.001)$. There were also group differences in SE-VEG scores based on children's perceptions of FV opportunities during after-school $(\mathrm{F}(1,176)=6.46, \mathrm{P}=$. 01). Similar to SE-FRUIT, children perceiving FV opportunities during after-school were significantly higher on SE-VEG compared to children not perceiving FV opportunities.

In addition to self-efficacy, differences emerged regarding children's proxy efficacy. Specifically, children in schools with low racial/ethnic diversity and higher-SES were significantly greater on PEFV-P than children in schools with high racial/ethnic diversity and lower-SES $(\mathrm{F}(1,176)=5.44, \mathrm{P}=.02)$. Moreover, children who perceived that their after-school environments provided more FV opportunities, were significantly greater on PEFV-S compared to youth not perceiving FV opportunities after-school (F (1, 176) $=25.46, \mathrm{P}=.0001)$.

\section{Discussion}

The current study supports the global hypothesis that self-efficacy and proxy efficacy are separate but related constructs within the FV context. The 14-item measure had two self-efficacy subscales and two proxy efficacy subscales. The measure demonstrated impressive factorial and criterion validity, as well as acceptable reliability among late elementary-aged children. Contrary to previous studies reporting self-efficacy and proxy efficacy as a one-dimensional construct, ${ }^{9}$ the present measure is consistent with SCT, hypothesizing that children's beliefs for personal agency and proxy agency are based on distinct skills and abilities. ${ }^{17}$

Two subscales measured children's self-efficacy for FVC, one self-efficacy scale for fruit consumption and another for vegetable consumption. This finding is consistent with previous research revealing that fruit consumption and vegetable consumption are 
independent behaviors. ${ }^{10-12}$ For example, Reinaerts and colleagues ${ }^{10}$ found the habitual eating behavior among 4-12-year old children explained $13 \%$ of the variance for their fruit consumption, but only $3 \%$ of the variance for their vegetable consumption. ${ }^{11}$ Additionally, Gibson et $\mathrm{al}^{12}$ discovered children's consumption of fruits are related to different psychosocial and environmental factors compared to their consumption of vegetables. These results demonstrate that FVC are different behaviors involving different antecedents; thus, supporting the present employment of separate self-efficacy measurements for each.

Similar to self-efficacy, two separate scales for proxy efficacy were established. One subscale captured children's proxy efficacy to influence parents and another concerned their confidence to influence after-school staff. This finding supports our hypothesis, that children's proxy efficacy varies depending on the authority figure in control of the environmental opportunities for FV (parents versus after-school staff). This finding may contribute to a future explanation for why children's FVC during the weekday at school-lunch differs from FVC at home. ${ }^{18-20}$

Criterion validity analyses provided further validity for the current measures. The hypothesis that self-efficacy for consumption of both fruits and vegetables would vary across groups was supported. Specifically, those children perceiving FV opportunities in after-school had higher self-efficacy for consuming fruit, higher self-efficacy for consuming vegetables and higher proxy efficacy for influencing after-school staff compared to children who did not perceive FV opportunities during after-school. This suggests that children's perceptions of FV opportunity in after-school may influence their self-efficacy and proxy efficacy, verifying adequacy of the current measure and its ability to capture and distinguish these differences.

Another finding emerging from the current analyses highlights differences in proxy efficacy at the school level. In the present study, the hypothesis that proxy efficacy will vary differently across school classification (diversity and SES) was supported. Specifically, those children attending lower diversity and higher-SES schools were significantly more confident they could influence their parents to make FV more available compared to children attending schools with higher racial/ethnic diversity and lower-SES. Racial/ethnic diversity and lower-SES are expected influences of FV 
availability; therefore, the distinction uncovered in this analysis further supports the criterion validity of the current measure and its use in future studies.

The present study offers several specific contributions, extending the FV research literature. There is limited research investigating the direct personal agency and proxy efficacy constructs, and even fewer evaluating measurement of these constructs. The present study not only extends understanding of specific efficacy constructs within the FV context, it also offers a reliable and valid measurement tool that may be applied in future research. Additionally, the measurement evaluation is strengthened with the inclusion of two types of validation as well as two types of reliability tests. Specifically, construct validity (factor analysis) unveiled four measurement constructs that also demonstrated appropriate criterion validity. Furthermore, appropriately high internal consistency was confirmed using both Cronbach's $\alpha$ coefficients and equal-length Spearman-Brown coefficients.

Along with strengths of the present research, the following limitations should also be noted. First, the sample may not represent the national population of elementary-aged children, but does include ethnic variability common in Kansas' public schools. Future research needs to test these self-efficacy constructs in more diverse samples and varying age groups to determine how self-efficacy develops over time, and how FVC is impacted. Secondly, the PEFV-P and PEFV-S subscales did not ask separate questions distinguishing fruit availability from vegetable availability. It may be possible that children's proxy efficacy from staff or parents varies dependent on whether they are requesting fruit versus requesting vegetables. Additionally, test-retest reliability (stability reliability) should be assessed for the scales.

In conclusion, the measure of self-efficacy for FVC and proxy efficacy for FV availability demonstrated acceptable factorial validity, reliability, and criterion validity in late elementary-aged youth. Results illuminate four valid constructs within the FV context, contributing a better understanding of the separate influences of self-efficacy and proxy efficacy. Future investigations are needed to determine if the self-efficacy and proxy efficacy constructs are central variables of the causal process determining changes in children's FVC. Development and evaluation of interventions aimed at increasing children's FVC may be one way to examine this question. Specifically, interventions 
targeting self-efficacy for FVC and proxy efficacy for FV availability can examine whether these variables mediate effectiveness of the intervention. Because self-efficacy for fruit consumption is separate from self-efficacy for vegetable consumption, interventions may need to consider separate strategies for improving each. Additionally, proxy efficacy for parents is a separate construct from proxy efficacy for after-school staff; thus, interventions may also need to consider separate strategies for increasing proxy efficacy for FV availability at home versus in the after-school environment.

\section{Acknowledgements}

This paper is based upon work supported by the Cooperative State Research, Education, and Extension Service, U.S. Department of Agriculture, under Award No. 2005-35215-15418.

We thank Karla Bruggeman, Tanis Hastmann and Sara Rosenkranz for assisting in data collection and data entry. 


\section{References}

1. Bandura A. Social foundations of thought and action: A social cognitive theory. Englewood Cliffs, NJ: Prentice-Hall; 1986.

2. Domel BS, Thompson WO, Davis M, Baranowski T, Leonard S, Baranowski J. Psychosocial predictors of fruit and vegetable consumption among elementary school children. Health Ed Research. 1996;11(3):299-308.

3. Kratt P, Reynolds K, Shewchuk R. The role of availability as a moderator of family fruit and vegetable consumption. Health Educ Behav. 2000;27:471-482.

4. Reynolds KD, Hinton AW, Shewchuk R., Hickey, CA. Social cognitive model of fruit and vegetable consumption in elementary school children. J Nutr Ed. 1999;31(1):23-30. 5. Reynolds KD, Yaroch AL, Franklin FA, Maloy J. Testing mediating variables in a school-based nutrition intervention program. Health Psychol. 2002;21(1):51-60.

6. Cullen K.W., Bartholomew L.K., Parcel G.S., Koehly L. Measuring stage of change for fruit and vegetable consumption in 9-to 12-year-old-girls. J Beh Med, 1998;21(3):241-254.

7. Bandura A. Social cognitive theory: An agentic perspective. Ann Rev Psychol. 2001;52:1-26.

8. Bandura A. (2000). Exercise of human agency through collective efficacy. Curr Directions Psychol Sci. 2000;9:75-78.

9. Reynolds KD, Yaroch AL, Franklin FA, Maloy J. Testing mediating variables in a school-based nutrition intervention program. Health Psychol. 2002;21(1):51-60.

10. Reinaerts E, Nooijer J, Candel M, Vries N. Explaining school children's fruit and vegetable consumption: The contributions of availability, accessibility, exposure, parental consumption and habit in addition to psychosocial factors. Appetite. 2006;48:248-258. 11. Vereecken CA., Van Damme W, Maes L. Measuring attitudes, self-efficacy, and social and environmental influences on fruit and vegetable consumption of 11 - and 12year-old children: reliability and validity. J Am Diet Assoc. 2005;105(2):257-261. 12. Gibson EL, Wardle J, Watts, CJ. Fruit and vegetable consumption, nutritional knowledge and beliefs in mothers and children. Appetite. 1998;31:205-22 
13. U.S. department of Health and Human Services (USDA). Dietary guidelines for Americans (6th ed.), Washington, DC: US Government Printing Office; 2005.

14. Kaiser H. A note on Guttmann's lower bound for the number of common factors. Multivariate Behavioral Research. 1961;1:249-276.

15. Cattell RB. The scree test for the number of factors. Multivariate Behavioral Research. 1966;3:245-276.

16. Gorsuch RL. Factor analysis. In Hillsdale, NJ: Lawrence Erlbaum Associates, Inc. (Ed.), (2nd ed.); 1983.

17. Bandura, A. (1997). Self-efficacy: The exercise of control. New York: W. H. Freeman.

18. Domel S, Baranowski T, Davis H, et al: Development and evaluation of a school intervention to increase fruit and vegetable consumption among 4th and 5th grade students. J Nutr Educ. 1993;25:345-349.

19. Luepker RV, Perry CL, McKinlay SM, et al: Outcomes of a field trial to improve children's dietary patterns and physical activity: The Child and Adolescent Trial for Cardiovascular Health (CATCH). JAMA. 1996;275:768-776.

20. Baranowski T, Davis M, Resincow K, et al: Gimme 5 fruit, juice, and vegetables for fun and health: Outcome evaluation. Health Educ Behav. 2000;27(1):96-111. 
Figures and Tables

Table 0-1 Exploratory Factor Analysis Results and Factor Loadings for FV Self-Efficacy and Proxy Efficacy Scales

\begin{tabular}{|c|c|c|c|c|c|}
\hline \multirow{2}{*}{ Factor Label } & \multirow{2}{*}{ Items } & \multicolumn{4}{|c|}{ Factor Loadings } \\
\hline & & 1 & 2 & 3 & 4 \\
\hline \multirow{3}{*}{ 1. SE-Vegetable } & One serving ( $1 / 2$ cup) of vegetables each day & .672 & .004 & .162 & .048 \\
\hline & Two serving ( $1 / 2$ cup) of vegetables each day & .914 & .050 & -.063 & -.095 \\
\hline & Three serving ( $1 / 2$ cup) of vegetables each day & .695 & .038 & -.083 & -.264 \\
\hline \multirow{4}{*}{ 2. PEFV-School } & Get the after-school staff to offer dried fruit snacks (like raisins, banana chips and apricots & -.013 & .733 & -.012 & -.018 \\
\hline & Get the after-school staff to offer applesauce cups or fruit cups (like fruit cocktail) & .038 & .823 & -.040 & .158 \\
\hline & Get the after-school staff to offer fruit and vegetable snack options & -.012 & .692 & .112 & -.005 \\
\hline & Get the after-school staff to offer $100 \%$ real fruit juice & .019 & .589 & -.042 & -.099 \\
\hline \multirow{4}{*}{ 3. PEFV- Parents } & Get your parents to buy fruit for snacks & .063 & -.080 & .711 & .045 \\
\hline & Get your parents to fix your favorite vegetable dish & .238 & .003 & .607 & .152 \\
\hline & Get your parents to keep $100 \%$ juice in the refrigerator & -.037 & .075 & .495 & -.234 \\
\hline & Get your parents to fix a fruit and vegetable snack & -.184 & .106 & .766 & -.139 \\
\hline \multirow{3}{*}{ 4. SE-Fruit } & One serving ( $1 / 2$ cup) of fruit each day & .151 & .076 & .088 & -.581 \\
\hline & Two serving ( $1 / 2$ cup) of fruit each day & .071 & .026 & .040 & -.767 \\
\hline & Three serving ( $1 / 2$ cup) of fruit each day & .028 & -.061 & -.018 & -.808 \\
\hline Eigenvalues & & 4.23 & 2.12 & 1.81 & 1.38 \\
\hline$\%$ Percentage & & 30.19 & 15.15 & 12.94 & 9.85 \\
\hline Cumulative \% & & 30.19 & 45.34 & 58.28 & 68.13 \\
\hline
\end{tabular}


Table 0-2 Group Leas Square Means and Standard Errors for FV Self-Efficacy and FV Proxy Efficacy

\begin{tabular}{|c|c|c|c|c|}
\hline \multirow[t]{2}{*}{ Group } & \multicolumn{2}{|c|}{ Self-Efficacy } & \multicolumn{2}{|c|}{ Proxy Efficacy } \\
\hline & Fruit & Vegetable & Parent & Staff \\
\hline \multicolumn{5}{|l|}{ Gender } \\
\hline Male $(n=97)$ & $2.65 \pm 0.06$ & $2.48 \pm 0.08$ & $2.60 \pm 0.06$ & $2.10 \pm 0.08$ \\
\hline Female $(n=89)$ & $2.56 \pm 0.06$ & $2.39 \pm 0.08$ & $2.53 \pm 0.06$ & $2.11 \pm 0.08$ \\
\hline \multicolumn{5}{|l|}{ Ethnicity } \\
\hline Diverse $(\mathrm{n}=53)$ & $2.62 \pm 0.08$ & $2.45 \pm 0.10$ & $2.59 \pm 0.08$ & $2.14 \pm 0.10$ \\
\hline White $(n=132)$ & $2.59 \pm 0.05$ & $2.41 \pm 0.07$ & $2.54 \pm 0.05$ & $2.07 \pm 0.07$ \\
\hline \multicolumn{5}{|l|}{ Household SES } \\
\hline Not Eligible $(\mathrm{n}=109)$ & $2.58 \pm 0.07$ & $2.38 \pm 0.08$ & $2.55 \pm 0.07$ & $2.05 \pm 0.09$ \\
\hline Eligible $(n=73)$ & $2.63 \pm 0.06$ & $2.48 \pm 0.08$ & $2.58 \pm 0.07$ & $2.16 \pm 0.08$ \\
\hline \multicolumn{5}{|l|}{ Weight Status } \\
\hline Normal $(n=141)$ & $2.52 \pm 0.05$ & $2.40 \pm 0.06$ & $2.50 \pm 0.05$ & $2.13 \pm 0.07$ \\
\hline At Risk/Overweight ( $\mathrm{n}=43$ ) & $2.69 \pm 0.08$ & $2.46 \pm 0.10$ & $2.63 \pm 0.09$ & $2.09 \pm 0.10$ \\
\hline \multicolumn{5}{|l|}{ Perceived School FV Opportunity } \\
\hline Opportunity $(\mathrm{n}=77)$ & $2.77 \pm 0.07 *$ & $2.55 \pm 0.08^{*}$ & $2.56 \pm 0.07$ & $2.34 \pm 0.09 *$ \\
\hline No-Unsure (n=109) & $2.44 \pm 0.06$ & $2.31 \pm 0.07$ & $2.57 \pm 0.07$ & $1.87 \pm 0.07$ \\
\hline \multicolumn{5}{|l|}{ School Diversity-SES } \\
\hline High Div.-Low SES & $2.53 \pm 0.07$ & $2.35 \pm 0.08$ & $2.45 \pm 0.07 *$ & $2.17 \pm 0.09$ \\
\hline Low Div.-High SES & $2.68 \pm 0.07$ & $2.51 \pm 0.09$ & $2.68 \pm 0.07$ & $2.04 \pm 0.10$ \\
\hline
\end{tabular}

Note: *P 